lichkeitserscheinungen ebensogut als $\mathrm{R}$ ot $\mathrm{h}$ munds Formel und stimmt ferner auch noch mit den Vorgängen bei der Löslichkeit von Hydraten, welche der Rothmundschen Formel widersprechen. Man muB nicht notwendig Abweichungen vom $\mathrm{D}$ al to $n$ schen Gesetz annehmen, um diese Löslichkeitsbeeinflussungen bei Anhydriden zu erklären, denn sie ergeben sich mathematisch aus der gewöhnlichen Theorie verdünnter Lösungen als die Folge der Hydra- tationsverschiebung der gelösten Substanzen, welche der Zusatz fremder Substanzen zur Lösung hervorruft. Diese Hydratationstheorie sagt keine gegenseitigen Löslichkeitsbeziehungen voraus, wie Rothmunds Formel, und erklärt die Löslichkeitswirkungen durch eine ganz andere Ursache, nämlich durch die Existenz von Hydraten in der Losung.

Washington D. C.

(Eingegangen: 4. November.)

\title{
VERGLEICHENDE UNTERSUCHUNGEN ÜBER SALZBILDUNG UND ÜBER DIE BASIZITÄT DER SÄUREN VOM PHYSIKO-CHEMISCHEN STANDPUNKTE.
}

Dritte Mitteilung ${ }^{1}$ ).

Von G. Bruni und C. Sandonnini.

\section{Prüfung der Minimumgleichung bei schwachen Sauren und konzentrierten Lösungen.}

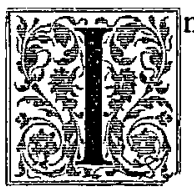

der zweiten Mitteilung hat der eine von uns eine allgemeine Gleichung abgeleitet, welche es gestattet, den Verlauf der Leitfähigkeitskurven der Säuren während der Neutralisation vorauszusehen und zu berechnen. Die Prüfung dieser Gleichung mit den experimentellen Resultaten ergab eine befriedigende Ueberein: stimmung. Nur ein Punkt sollte noch aufgeklärt werden.

Oben erwähnte Gleichung sieht für den Fall, daß die freie Säure ein kleineres Leitvermögen als das neutrale Salz besitzt, immer das Vorhandensein eines Minimumpunktes voraus, welcher sich mit steigender Energie der Säure und mit wachsender Verdünung von der Säureseite nach der Salzseite verschiebt.

Die Prüfung dieser Forderung der Theorie war bei der ersten Versuchsreihe nicht vollständig durchgeführt worden, insofern, als bei der schwächeren Essig- und Ameisensäure für die konzentrierteren Lösungen keine Minima direkt festgestellt worden waren. Für die Essigsäure trat das Minimum bei den bisher veröffentlichten Versuchsergebnissen erst für $V$ $=256$ ein, für Ameisensäure erst bei $V=8$.

Schon damals ${ }^{2}$ ) wurde aber betont, daß die Ursache hiervon blo $B$ in dem Umstand zu suchen war, da $\beta$ die fragliche Minima, wie sie sich aus der Gleichung berechnen lassen, zu dicht an der Săureseite liegen sollten und somit bei den damaligen Versuchsbedingungen nicht festgestellt werden konnten. Bei Essigsäure waren nämlich zwischen 0,0 und 0,05 Molen Base pro

I) I. u. II., G. Bruni, Z. f. Elektroch. 14, 7ot, 729 (1908).

2) 1. c., S. $73^{\mathrm{r}}$.
Mol Säure keine Bestimmungen ausgeführt worden, während alle fragliche Minima nach der Theorie sich in diesem Neutralisationsintervall befinden sollten.

$Z_{\text {weck }}$ vorliegender Arbeit war es, diesen Punkt experimentell eingehend zu prüfen. Wir haben somit mit Essigsăure bei Verdünnungen zwischen $V=\mathrm{I}$ und 256, mit Ameisensăure zwischen $V=\mathrm{I}$ und 8 experimentiert, und zwar mit Zusatz kleinerer Mengen Alkali. Ferner haben wir mit einer noch schwächeren Säure gearbeitet, nämlich mit $p$-Nitrophenol, deren Dissoziationskonstante nach $\mathrm{Bade}{ }^{1}$ ) $K=$ $0,000012 \cdot 10^{-2}$, nach Hantzsch $\left.{ }^{2}\right) K=96 \cdot 10^{-7}$ ist. Mit letzterer Säure hatten schon Thiel und Roemer (l.c.) Messungen ausgeführt, ohne, aus den früher angeführten Gründen, ein Minimum zu finden. Hier sind Bestimmungen zwischen $V=128$ und 1024 ausgeführt worden, und anstatt $\mathrm{KOH}$ wurde $\mathrm{NaOH}$ angewandt.

$\mathrm{Da}$ es sich hier um die Feststellung ziemlich kleiner Differenzen in dem Leitungsvermögen handelte, so ist auf die sehr genaue Bereitung der Lösungen besonderes Gewicht gelegt. Die teilweise Neutralisation der Säure ist zu diesem Zweck über einem größeren Volumen der Flüssigkeit ausgeführt worden. Ein praktisches Beispiel hiervon sei hier angegeben. $\mathrm{Zu} 500 \mathrm{ccm}$ $z$ weifach normaler Essigsäure wurden $3 \mathrm{ccm}$ normaler $K O H$-Lauge hinzugefügt und das Ganze zu I Liter verdünnt; man erhielt somit eine Lősung, die I Mol Säure und o,003 Mole Alkali enthält, usw. Die Messung wurde dänn, wie immer, mit $20 \mathrm{ccm}$ dieser Lösung ausgeführt.

Für eine größere Reinheit des Wassers wurde auch gesorgt; die betreffende Eigenleitfähigkeit betrug ungefähr $3 \cdot 10^{-6}$ und wurde,

I) Zeitschr. f. physik. Chemie 6, 297 (I890).

2) Berichte 32, III, 3070 (I899). 
wo nötig, von der Gesamtleitfăhigkeit subtrahiert.

Die erhaltenen Resultate sind aus den folgenden Tabellen und Figuren ohne weiteres ersichtlich.

Tabelle I (siehe Fig. 424).

A meisensäure $+\mathrm{KOH}$. $\left.(K=0,00020)^{1}\right)$.

\begin{tabular}{|c|c|c|c|c|c|}
\hline & $\begin{array}{c}\text { Molen } \\
K O H \text { auf } \\
\text { r Mol Suure }\end{array}$ & $V=\mathrm{I}$ & a & 4 & 8 \\
\hline 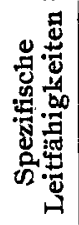 & $\begin{array}{l}0,0 \\
0,01 \\
0,02 \\
0,03 \\
0,04 \\
0,05 \\
0, I\end{array}$ & $\begin{array}{c}\left.5,8^{2}\right) \\
5,45 \\
5,45 \\
5,87 \\
6,56 \\
7,3 \\
17,7\end{array}$ & $\begin{array}{l}3,95 \\
3,85 \\
3,76 \\
3,86 \\
4,13 \\
4,44 \\
6,52\end{array}$ & $\begin{array}{l}2,75 \\
2,71 \\
2,64 \\
2,56 \\
2,67 \\
2,82 \\
3,67\end{array}$ & $\begin{array}{l}2,05 \\
1,91 \\
1,85 \\
1,81 \\
1,84 \\
I, 86 \\
2, I 6\end{array}$ \\
\hline 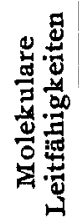 & $\begin{array}{l}0,0 \\
0,01 \\
0,02 \\
0,03 \\
0,04 \\
0,05 \\
0,1\end{array}$ & $\begin{array}{c}5,80 \\
5,45 \\
5,45 \\
5,87 \\
6,56 \\
7,3 \\
r r, 7\end{array}$ & $\begin{array}{r}7,90 \\
7,70 \\
7,52 \\
7,72 \\
8,26 \\
8,88 \\
13,04\end{array}$ & $\begin{array}{l}I I, 00 \\
I 0,85 \\
10,56 \\
I 0,24 \\
I 0,68 \\
I I, 28 \\
I 4,68\end{array}$ & $\begin{array}{l}I 6,40 \\
I_{5,28} \\
I_{4}, 80 \\
I_{4,48} \\
I_{4,72} \\
I 4,88 \\
I 6,96\end{array}$ \\
\hline
\end{tabular}

I) Wahre Werte.

a) Die kursiv gedruckten Zahlen sind aus den fruheren Bestimmungen entnommen.

Wie man sieht, ist die erste Forderung der Theorie, nämlich das Vorhandensein der Minima und ihre Verschiebung nach rechts mit steigender Verdünnung, uberall erfült. Wir wollen jetzt untersuchen, wie die Lage der Minima mit den theoretisch berechneten übereinstimmt. Die Be- rechnung geschieht, wie in der zweiten Abhandlung, durch die Gleichung:

$$
\begin{gathered}
x_{\text {min. }}=K V+\frac{2 A}{\sqrt{\mathrm{I}-A^{2}}} \cdot \sqrt{K V}, \\
A=\mathrm{r}-2 \frac{l_{n}+l_{A}}{l_{H}+l_{A}} .
\end{gathered}
$$

wo

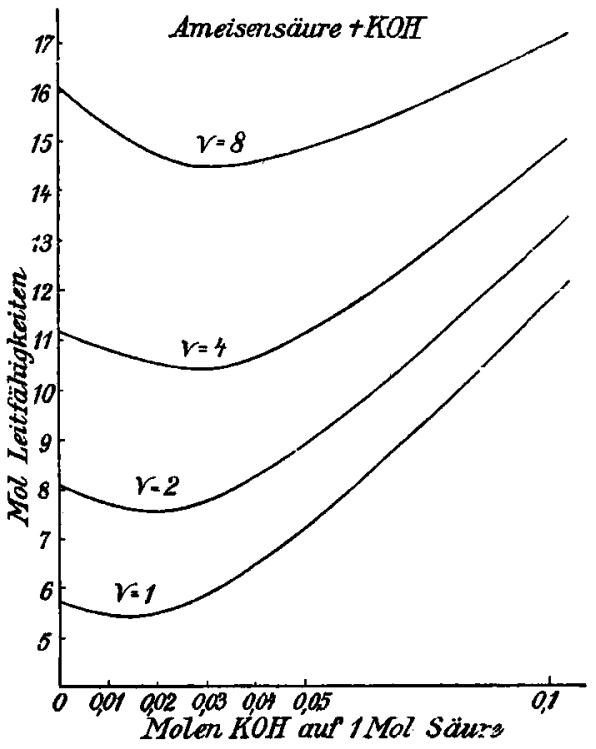

Fig. 424 .

Für Ameisensäure und Essigsäure wurden für $K$ und $A$ dieselben Zahlenwerte wie früher angewandt. Für $p$-Nitrophenol folgt aus unseren Bestimmungen: $l_{n}+l_{A}=9 \mathrm{r}$, wodurch $l_{H}+l_{A}$ $=390 ;$ ferner $K$ als Mittelwert 0,000000I,

Tabelle 2 (siehe Fig. 425). Essigsăure $+\mathrm{KOH}$.

$(K=0,000017$. $)$

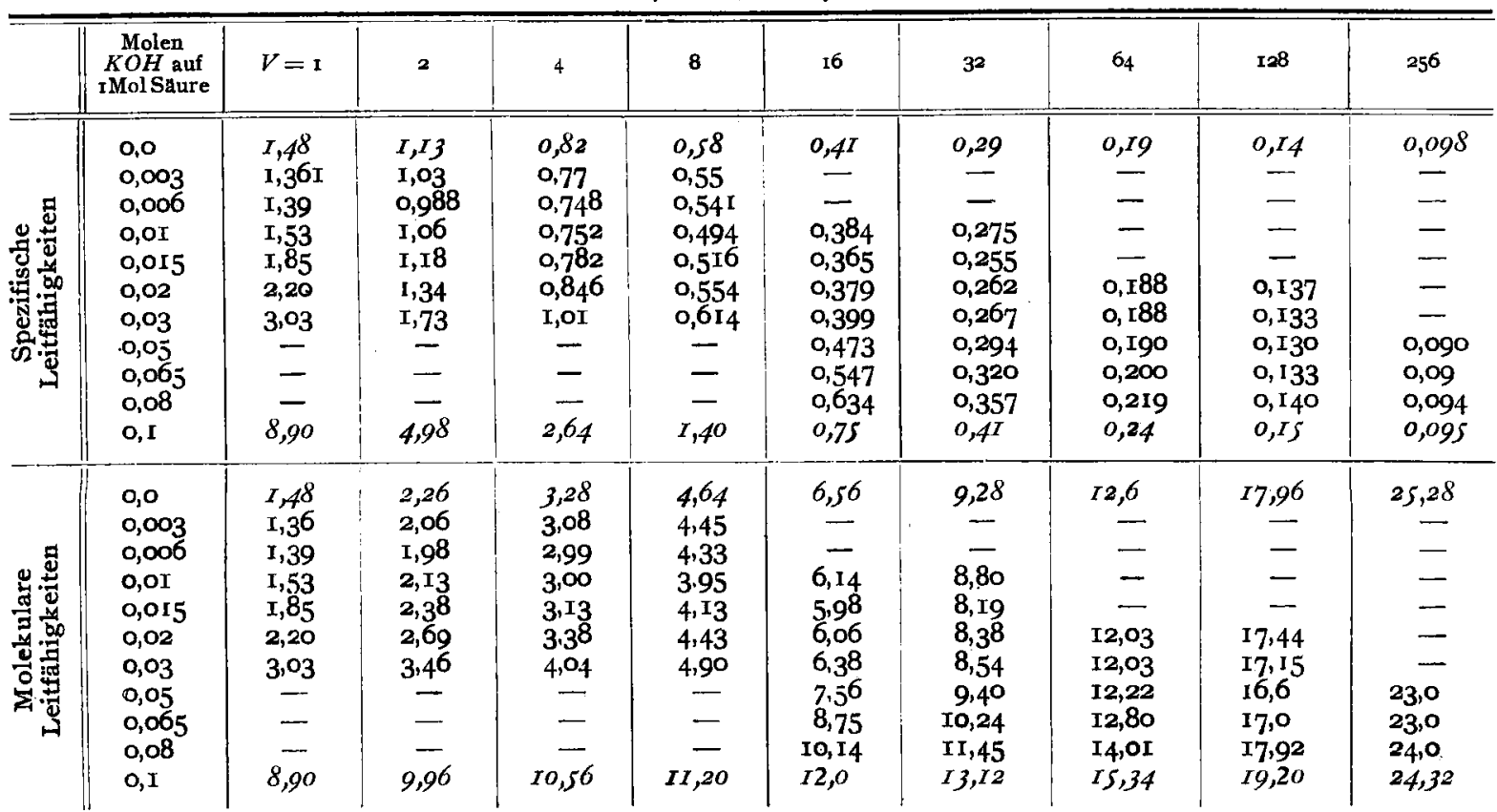


während Bader (1. c.) 0,000000 I 2 und Hantzsch (l. c.) 0,000000096 angeben.

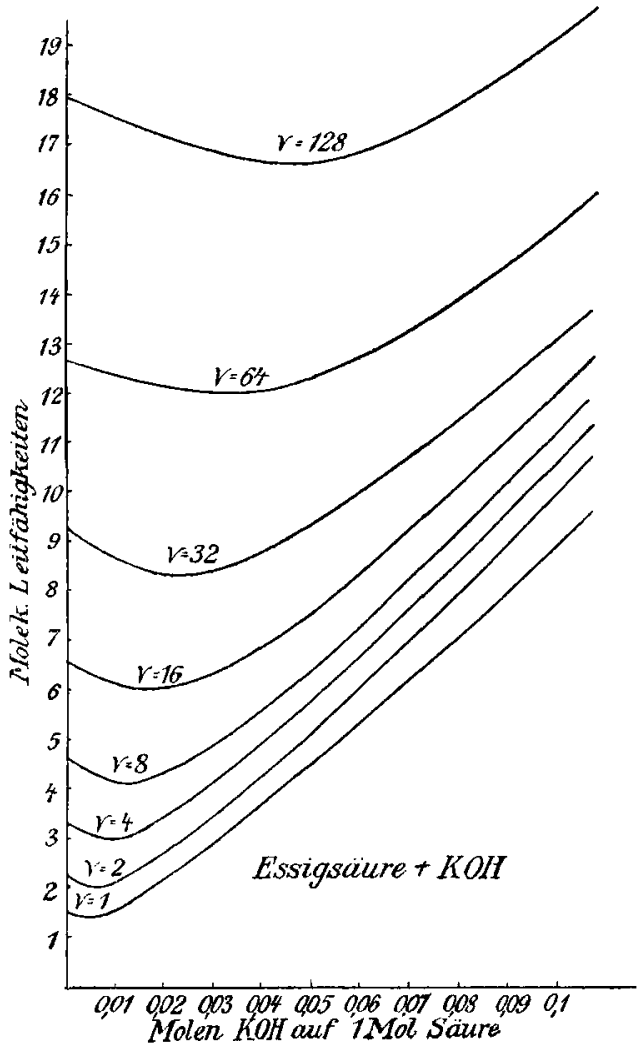

Fig. 425 .

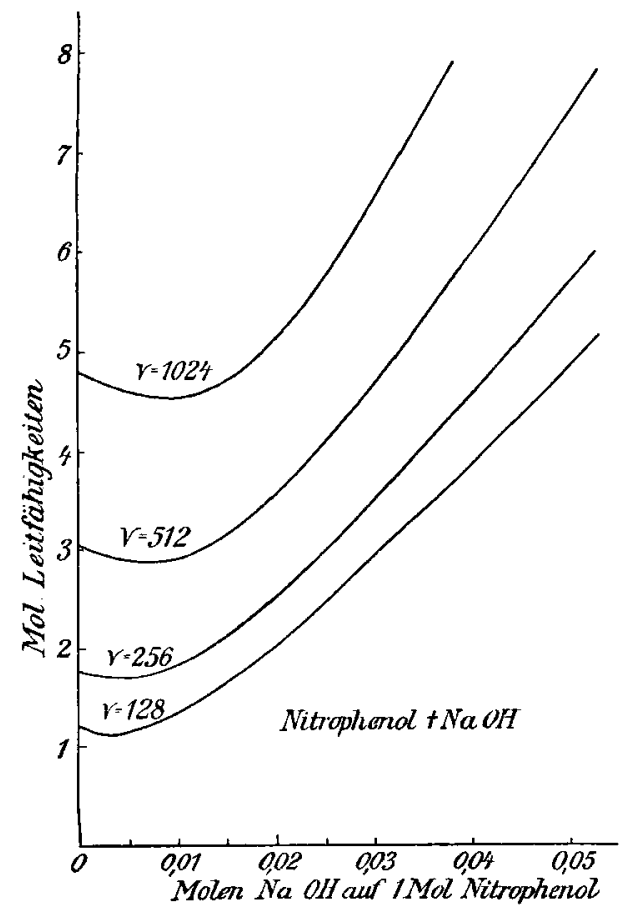

Fig. $4^{26 .}$
Tabelle 3 (siehe Fig. 426).

$p$-Nitrophenol $+\mathrm{NaOH}^{1}$ ).

( $K=$ cooooor. $)$

\begin{tabular}{|c|c|c|c|c|c|}
\hline & $\begin{array}{c}\text { Molen } \\
\text { NaOH auf } \\
\text { i Mol Saure }\end{array}$ & $V=\mathrm{t} 28$ & $25^{6}$ & $5^{12}$ & 1024 \\
\hline \multirow{11}{*}{ 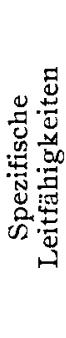 } & 0,0 & 0,0095 & 0,0068 & 0,0057 & 0,0046 \\
\hline & 0,005 & 0,0092 & 0,0067 & 0,0056 & 0,0045 \\
\hline & $0, O I$ & 0,0107 & $0,007 \mathrm{I}$ & 0,0056 & 0,0044 \\
\hline & 0,015 & 0.0129 & 0,0082 & 0,0066 & 0,0047 \\
\hline & 0,02 & $0,015^{6}$ & 0,0099 & $0,007^{2}$ & $0,005 \mathrm{I}$ \\
\hline & 0,05 & 0,0378 & 0,0222 & 0,0145 & 0,0100 \\
\hline & 0,125 & 0,0853 & 0,0465 & 0,0277 & 0,0175 \\
\hline & 0,25 & 0,167 & 0,0861 & 0,0487 & 0,0270 \\
\hline & 0,5 & $0,3^{18}$ & 0,170 & $0,090^{\circ}$ & 0,0492 \\
\hline & 0.75 & 0,473 & 0,249 & 0,130 & 0,0692 \\
\hline & $\mathrm{I}, \mathrm{O}$ & 0,610 & 0,325 & 0,169 & 0,0877 \\
\hline \multirow{11}{*}{ 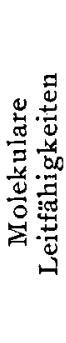 } & 0,0 & $\mathrm{I}, \mathbf{2 I}$ & $I, 76$ & 3,02 & 4,78 \\
\hline & 0,005 & $\mathrm{I}, 15$ & I.7 I & 2,87 & $4,5^{6}$ \\
\hline & 0, or & 1,36 & $\mathbf{I}, \mathbf{8} \mathbf{r}$ & 2,89 & 4,50 \\
\hline & 0,015 & $1, \overline{6}_{5}$ & 2,09 & 3,18 & $4,8 \mathrm{r}$ \\
\hline & 0,02 & $\mathbf{I}, 9 \overline{8}$ & 2,57 & 3.59 & 5,13 \\
\hline & 0,05 & 4,83 & 5,70 & $7.4^{\circ}$ & 10,2 \\
\hline & 0,125 & 10,9 & II, 9 & 14,19 & 17,9 \\
\hline & 0,25 & 21,3 & 22,0 & 24,9 & 275 \\
\hline & 0,5 & 40,6 & 43.4 & 46,0 & 50,3 \\
\hline & 0,75 & 60,5 & 63,7 & 66,5 & 70,8 \\
\hline & $I, O$ & 78,0 & 83,3 & 86,5 & 89,8 \\
\hline
\end{tabular}

Tabelle 4 .

\begin{tabular}{|c|c|c|c|c|c|c|c|c|}
\hline \multicolumn{3}{|c|}{ Ameisensăure } & \multicolumn{3}{|c|}{ Essigsăure } & \multicolumn{3}{|c|}{$p$-Nitrophenol } \\
\hline$V=$ & $\underset{\text { ber. }}{x_{\min }}$ & $\underset{\text { gef. } 1)}{x \text { min. }}$ & $V=$ & $\underset{\text { ber. }}{x_{\text {min }}}$ & $x_{\text {mefin. }}$ & $\nabla=$ & $\underset{\text { ber. }}{x_{\min }}$ & $x_{\min }$ \\
\hline I & O,OI I & 0,013 & I & 0,0037 & 0,004 & 123 & 0,0049 & 0,004 \\
\hline 2 & 0,016 & 0,012 & 2 & 0,0053 & 0,006 & 256 & 0,007 & 0,005 \\
\hline 4 & 0,023 & 0,027 & 4 & 0,008 & 0,009 & 512 & O,OIO & $0,00 \overline{8}$ \\
\hline \multirow[t]{5}{*}{8} & $0,03^{2}$ & $0,03^{2}$ & 8 & $0,01 \mathrm{I}$ & 0,012 & $\mathrm{rO}_{4}$ & $0,0 \mathrm{I}_{4}$ & 0,010 \\
\hline & & & 16 & 0,015 & 0,015 & & & \\
\hline & & & $3^{2}$ & 0,022 & 0,022 & & & \\
\hline & & & $6_{4}$ & 0,032 & $0,03^{2}$ & & & \\
\hline & & & I 28 & 0,045 & 0,044 & & & \\
\hline
\end{tabular}

I) Graphisch interpolierte Werte.

Man sieht, daß auch hier die Uebereinstimmung, besonders für die zwei ersteren Säuren, eine ganz ausgezeichnete ist.

Weitere Untersuchungen sind im Gang.

Padua, Institut für allgemeine Chemie der Universität, November I9o8.

(Eingegangen: 20. November.)

I) Unsere Zahlen sind mit denjenigen von $T h i \mathrm{iel}$ und Roemer nicht direkt vergleichbar. Bei diesen Autoren wächst einerseits das Volumen von 75 bis 85 ; anderseits enthalten ihre Lösungen immer Io $\mathrm{ccm}$ Alkohol, welcher auf das Leitvermögen einen erheblichen Einfluß ausüben soll. Mit denjenigen von Hantzsch (1.c.) stimmen unsere Resultate gut überein. 